\section{Interview with Dr José M Zuniga}

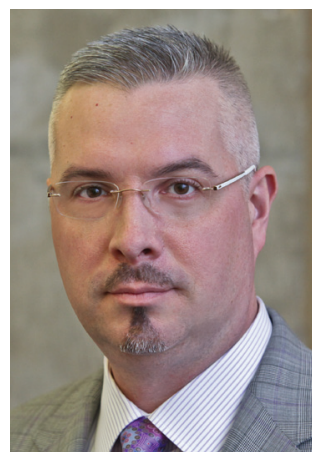

José M Zuniga* speaks to Frances Adlam, Commissioning Editor: Dr José M Zuniga was appointed President of the International Association of Providers of AIDS Care (IAPAC) in 1999. Prior to his appointment, he served as IAPAC's Vice President, charged with advancing the association's medical education and technical assistance initiatives. He lectures at several academic institutions; has been published in various journals; has authored thematic chapters for four books and edited three books: The HIV Pandemic (Oxford University Press [OUP], 2006); Decade of HAART (OUP, 2008) and Advancing the Human Right to Health (OUP, 2013). $\mathrm{He}$ is Editor of the Journal of the International Association of Providers of AIDS Care (JIAPAC). He is an IAPAC Trustee, and a member of the Joint United Nations Programme on HIV/AIDS (UNAIDS) Scientific and Technical Advisory Committee. He routinely advises United Nations health agencies, as well as bilateral institutions, such as the US President's Emergency Plan for AIDS Relief. He is a member of the American Public Health Association and the National Hispanic Medical Association.

First draft submitted: 16 June 2016; Accepted for publication: 25 July 2016; Published online: 15 August 2016

Q Can you tell us a little about your career to date \& what your current roles entail? I am a public health specialist by training, with a human development and social policy background. I joined the International Association of Providers of AIDS Care (IAPAC) 19 years ago, serving first as the association's Vice President and, subsequently, as President since 1999. My role today is to coordinate all of the association's various activities aimed at expanding access to and improving the quality of HIV testing, prevention, care and treatment services for people living with and affected by HIV/AIDS and its comorbid conditions. Our activities range from education to technical assistance, advocacy to research, as well as global health initiatives, such as the Fast-Track Cities initiative [1].

\section{Q Apart from Fast-Track Cities, what other projects has IAPAC been involved in?}

IAPAC has been engaged in capacity building from the very beginning of our existence, 30 years ago. Through the early years of the HIV epidemic, we were engaged in nutrition and palliative care because we had no, or few, HIV treatment options. As our options expanded we increased the scope and breadth of the capacity building we conducted worldwide, most notably leveraging the advent of antiretroviral therapy (ART) in regions of the world that were being excluded from the benefits of these life-saving and enhancing medications.

Over the past 3 years, our work has been restructured so that each activity contributes to an overall aim of controlling the HIV epidemic, including a hearty embrace of

*International Association of Providers of AIDS Care, 2200 Pennsylvania Avenue, NW, 4th Floor East, Washington, DC, 20037, USA; jzuniga@iapac.org

\section{KEYWORDS}

AIDS $\bullet$ HIV $\bullet$ prevention $\bullet$ treatment 
decisive targets, such as the UNAIDS 90-9090 targets [2]. In 2015, we launched our IAPAC Guidelines for Optimizing the HIV Care Continuum for Adults and Adolescents [3]. We are currently engaged in training across 16 sub-Saharan African countries through the IAPAC African Regional Capacity-Building Hub [4], building clinician capacity for managing HIV, as well as HBV and HCV coinfection. On the research front, we are involved in an NIH/President's Emergency Plan for AIDS Relief-funded study exploring how patientcentered care might facilitate increased HIV testing and optimized linkage to care in highHIV-burden settings. On the advocacy front, we are hosting our sixth annual summit this year focused on the political and practical aspects of controlling the HIV epidemic with ART, bringing together key opinion leaders from every relevant perspective - researchers, clinicians, government officials, donor agency representatives, civil society members and affected communities - to advocate the immediate wide-scale implementation of treatment as prevention (TasP) and pre-exposure prophylaxis (PrEP) in every region of the world. We do much, much more as an association of 27,000-plus providers of HIV services, but these are some of the programmatic highlights at the moment apart from our work with Fast-Track Cities.

\section{Q Can you explain what the UNAIDS 90- 90-90 targets are \& how the targets were decided? Are they achievable?}

Given a directive by UNAIDS' visionary Executive Director, Mr Michel Sidibé, the UNAIDS Scientific and Technical Advisory Committee, of which I am a member, conceived the 90-90-90 targets to jumpstart the global AIDS response in the face of scientific evidence of the preventative benefits of ART. Under the extraordinary leadership of its chair, Dr Julio Montaner of the British Columbia Centre of Excellence in HIV/AIDS, this committee of researchers, clinicians, public health specialists and modelers looked at and extrapolated available data to confidently posit that we could bend HIV morbidity and mortality curves and get to HIV epidemic control if we were to attain 90-90-90 targets by 2020 . That is $90 \%$ of people living with HIV/AIDS (PLHIV) knowing their HIV status, $90 \%$ of PLHIV who know their status on ART and $90 \%$ of PLHIV on ART achieving viral suppression.
Do I think the targets are attainable? Absolutely, but not without hard work. From looking at the data, we are noting that some cities and countries that are investing in HIV care continuum optimization are close to attaining at least one and in some instances two of the 90s and may be struggling with the other. Not surprisingly, cities and countries that have optimized their AIDS responses and/or where significant investments have been made in health system strengthening and other interventions are doing better than others. That is where capacitybuilding, data-driven decision-making, course corrections or innovative programming come in to play.

\section{Q Could you explain a little bit more about Fast-Track Cities?}

The Fast-Track approach was developed by UNAIDS as a means to accelerate the global response against HIV/AIDS through decisive targets [5]. The global community has pledged itself to attain 90-90-90 and zero stigma and discrimination targets which, based on UNAIDS-commissioned modeling, would represent an important step toward ending AIDS as a public health threat by 2030 . The $90-90-90$ targets require that $90 \%$ of people living with HIV know their HIV status, 90\% of PLHIV who know their status are on ART and 90\% of PLHIV on ART achieve viral suppression. IAPAC's approach is to maximize the impact of ART by optimizing the HIV care continuum, noting that there are barriers to ART scale-up that we need to systematically address, mostly on the HIV testing side. It also focuses on ensuring that HIV-infected people are engaged in care and adhere to ART so that they can achieve sustained viral suppression, without which you cannot decrease AIDS-related mortality and reduce HIV transmission rates. And, because stigma and discrimination pose significant barriers for affected communities to access and utilize the full range of HIV services, our approach includes a much-needed focus on measuring and eliminating stigma and discrimination.

It seems natural that we should focus our approach by using the weapon in our armamentarium that can have the most impact (HIV treatment), and that we also focus geographically where the majority of PLHIV reside. Globally, unsurprisingly, these geographic 'hot spots' are in large urban settings. In fact, in some cities around the world, up to $60 \%$ of PLHIV reside in 
cities, which is only expected to increase as global urbanization continues its upward trend. Thus, the Fast-Track Cities initiative overlays the FastTrack approach atop existing AIDS responses in cities that are highly HIV burdened and assists them to accelerate these responses to attain the 90-90-90 and zero stigma and discrimination targets by 2020 .

There are four core partners assisting Fast-Track Cities: IAPAC, UNAIDS, the United Nations Human Settlements Program (UN-Habitat) and the city of Paris. It is important to note that the city of Paris was the first city to sign the Paris Declaration on Fast-Track Cities Ending the AIDS Epidemic. We launched Fast-Track Cities on World AIDS Day 2014, in Paris, and they have been active as real leaders over the past 18 months. Paris' Mayor, Ms Anne Hidalgo, has been a great leader by example, demonstrating how a city can commit to and accelerate its local AIDS response and focus its existing programming and resources to attain the initiative's decisive targets. The city of Paris coordinated 17 different consultations with key affected populations and communities in the French capital to build consensus around then to strategize about how to reorient their HIV programs - from testing, to treatment, to prevention, to the ongoing support patients require to achieve viral suppression - notably among that city's key populations.

Various Fast-Track Cities have also been working with IAPAC to develop a monitoring and evaluation (M\&E) framework that we launched at the 21st International AIDS Conference (AIDS 2016 [1]). The use of webbased M\&E platforms will allow us to visualize HIV testing, ART coverage, and viral suppression data to demonstrate the progress of individual cities in reaching the 90-90-90 targets. Each Fast-Track City gets a dashboard, which is an important means by which we intend to guarantee the open and transparent use of data to ensure that city stakeholders and, indeed, the global partners engaged in helping cities, remain accountable to all other stakeholders, primarily affected communities.

\section{Q The initiative focuses on the impact of} HIV/AIDS in cities, what can be done to target the rural communities?

In some instances there are spillover opportunities for rural communities to benefit from strengthened urban communities and the experiences that they gain in optimizing the HIV care continuum. There is a need, obviously, to expand to our rural communities and it is something we will consider in the future. However for the sake of prioritization we had to look at where the highest level of disease burden lies, which is in the urban areas of most countries. At this time I think there will be more focus on sharing best practices, allowing for networking between stakeholders in urban and rural settings so that we showcase what works and what does not and foster a sense of solidarity toward our global objective of controlling the HIV epidemic. Ultimately, the cities are laboratories for experimentation and innovation - finding new ways of thinking about and approaching challenges, including those that affect key populations.

\section{Q How important is the idea of sharing} knowledge \& the use of 'open data' to reach the goals set out by Fast-Track Cities?

One of the challenges we faced early on is the fact that in many jurisdictions we were not measuring the right things - or measuring what matters to make informed decisions regarding mid- to long-term AIDS responses. However, the 90-90-90 targets forced us all to adopt a standardized metric for success and then go out into either countries or cities and assist them with defining their baseline numbers. That is the work that we are doing now in Fast-Track Cities. Amsterdam, Denver, Kyiv, and Paris announced their 90-90-90 data as of July 2016, and we expect many more cities to do so in the coming months. Because we need to demonstrate forward momentum toward attaining the 90-90-90 targets year after year, the embrace of open data is so important - numbers speak louder than words, particularly if they point toward success in getting more people tested, treated and virally suppressed in geographic 'hotspots' that, if left as is, prevent our ability to end AIDS as a public health threat.

It is important to embrace open data and its transparent use to remain accountable to stakeholders, at local, national, regional and international levels. With Fast-Track Cities particularly, there is a requirement for cities to facilitate the open and transparent use of data as part of the initiative's M\&E framework. This involves technical handshakes that happen between local health departments and IAPAC to ensure that data are generated and reported 
utilizing systematic 90-90-90 and HIV care continuum methodologies. But our work does not end with generating and reporting data. There is a lot of work that goes into addressing gaps that cities are identifying across their HIV care continua - all of it focused exactly where we can have the most impact, as well as among those specific populations most affected by HIV/AIDS.

While 90-90-90 are our primary targets, cities are at liberty to set additional targets. They may also establish locally relevant indicators. For example, in a number of countries around the world, PrEP has either been approved or will soon be approved for prescription to reduce HIV acquisition. There are therefore ongoing discussions with some Fast-Track Cities regarding how we track PrEP uptake. The use of shared data, particularly because it is geolocated within a city, will allow us to target PrEP education and support resources where it makes sense to do so. A Paris arrondissement that has low PrEP uptake yet where we project that a large percentage of people would benefit from PrEP is a strategically sound place for us to focus educational and support resources. This data-driven, geographic focus will result in PrEP uptake that translates into reduced HIV acquisition in that specific arrondissement where need has been documented and where our success can be measured through the city dashboards and by other means which the city elects to utilize. The bottom line is that we cannot focus our efforts and resources without data; without data we are blind.

\section{Q You have mentioned PrEP, what do you think are the most effective prevention \& treatment methods currently available?}

Data demonstrate that TasP can decrease the ability of PLHIV to transmit HIV by approximately $96 \%$, and that PrEP can reduce HIV acquisition by up to $92 \%$. There are questions regarding which intervention is more effective, clinically and cost-wise, that are being debated in the scientific literature. However, it is clear that both should be viewed within the context of combination HIV prevention, meaning combined with a variety of other interventions, such as condoms, voluntary male medical circumcision, postexposure prophylaxis and harm reduction strategies for substance users. From the IAPAC perspective, the attainment of the 90-90-90 targets is wholly dependent on our ability to get more people tested, to get those who are positive on ART, and to get those who are on ART virally suppressed. Of course, for those who test negative the linkage to preventative care is critical, including but not limited to PrEP, and should be viewed and actioned as a priority as we strive toward controlling the HIV epidemic.

Q How can we tackle the negative impact of discrimination of people with HIV/AIDS?

Stigma and discrimination pose significant barriers toward attaining the 90-90-90 targets, and it is why the HIV community insisted that UNAIDS also set zero stigma and discrimination targets. From a stigma perspective, we are focused on identifying the best interventions that will allow us to address the internalized stigmas experienced by people living with and affected by HIV. As important, though, we are working to mitigate stigmatizing communication in healthcare settings through sensitization and other types of training that will improve provider-patient communication and, by extension, break down barriers impeding access to and utilization of HIV services.

Equally important, though, is understanding that laws matter, that a care environment in which people feel legally discriminated against and even persecuted matters. Laws that criminalize the conduct of key populations create seemingly insurmountable barriers to people accessing HIV services, thus creating a situation in which people present in the later stages of HIV disease because they are afraid to get tested for HIV. Laws that criminalize perceived HIV exposure stigmatize people living with HIV/AIDS in ways that we are beginning to discern through HIV service utilization data, but that is simply the tip of the iceberg. We are not even close to understanding the full impact of laws that criminalize perceived HIV exposure, yet we are aware that in 61 countries around the world it has been prosecuted.

At the end of the day, though, well beyond the data is an incontrovertible truth that we must accept: stigmatizing and discriminating against people living with and affected by HIV/AIDS is a human rights violation. We can do everything else right, but if we fail to repeal laws that criminalize an entire population of people, or fail to address the stigma that denies an individual her/his dignity, than we are jeopardizing our potential success by also denying people the right to realize their right to health. 
Q In your opinion, what will be the main challenges in ending AIDS as a public health threat by 2030 ?

Attending the UN High-Level Meeting on HIV/AIDS in New York recently, I was encouraged to hear Minister of Health after Minister of Health embrace the 90-90-90 targets, the goal of ending AIDS as a public health threat by 2030 and the importance of leaving no one behind, notably key populations. Yet, the meeting's outcome - its political declaration [6] - exposed one of the greatest challenges we face, which is that we do indeed leave people behind. The political declaration did not speak as clearly it should have about the vulnerability of key populations to HIV/AIDS, thus rendering whole groups of people virtually invisible. These populations are where the highest burden of HIV disease lies, sometimes in countries that are hostile toward or, at best, not accommodating of men who have sex with men, transgender individuals, sex workers, and substance users. Language specific to key populations was stripped from the political declaration by countries on the wrong side of this human rights/public health issue. Thus, our challenge in this respect is that these individuals do not become invisible and therefore get driven underground in local, national, regional, and international AIDS responses.

Thankfully, we had our heroes at the UN High-Level Meeting on HIV/AIDS, including the US government, which created a US\$100 million new Key Populations Investment Fund specific to the implementation of interventions for key populations. But we need more than this generous gesture, and this takes me to the issue of resources. The resources necessary to get the job done have not yet been committed and so there are funding constraints that we are enduring as a community, both as a result of the economic downturn, but also the lack of political will to continue to invest in the fight against HIV/AIDS just as we have an opportunity to scale-up interventions that could help us to control the HIV epidemic. Political will that goes hand in hand with financial investments - both bilateral and multilateral - is critically needed now.

Finally, I would cite complacency and lack of accountability as significant challenges. We are in the 35th year of the HIV epidemic as of 5 June 2016. There is fatigue - we see this with donors who are rethinking their HIV/AIDS investments and we definitely see it with the media whose coverage of the HIV epidemic is increasingly anemic. Nonetheless, we need to maintain our momentum and I believe that the use of data will be incredibly helpful to us. For example, the day that we are able to say that $\mathrm{X}, \mathrm{Y}$ and $\mathrm{Z}$ cities or countries have attained the 90-90-90 targets, we can translate these successes - numbers of infections averted, numbers of AIDS-related deaths averted, less dollars spent on disease management - into a measurable sense of forward momentum toward ending AIDS as a public health threat. But that will all go by the wayside if we allow complacency to set in, leading to a failure to move the global HIV agenda toward its much-dreamed-of conclusion. Additionally, none of our aspirations can be realized without an embrace of the concept that we are all accountable to each other. At play here are elements of individual, community-wide, and population-level accountability. We cannot shy away from an accountability framework in which the individual, not the institution or the state, owns the process by which we define and enforce accountability and, ultimately, can leverage its outcomes to effect change.

\section{Q What do you see for the future of HIV/AIDS?}

That is a tough question to answer. I have been at this for a very long time, 19 years at IAPAC and several more years before that, meaning that I have seen such incredible progress that defines this unique HIV movement. Just think about the history of HIV from 'death sentence' in the early and mid-1980s, to a glimmer of hope in the early 1990s with mono and then dual ART, to the advent of ART in 1996. We have successfully transitioned HIV into a chronic condition, but only where we can guarantee people access to a full HIV care continuum, including but certainly not limited to ART.

The fact that we now have 17 million people on ART globally contrasted with a time when we had such nominal numbers of people on ART, mostly in the global North, is remarkable. That we accomplished such a feat against the backdrop of people saying 'Why bother expanding access to ART in the global South' for a number of insensible reasons, well, that is beyond remarkable and speaks to a refusal to live in a world with two standards of HIV care. To be clear, we still have unacceptable numbers of people not on ART. But given the pace of progress over the past 35 years, I have every expectation 
that we can close the gap and get to universal ART coverage within my lifetime. I am optimistic because we have a track record of success, we have a community that is passionate and tenacious, and we have clinical and other providers laboring with commitment and compassion. We are weary, but we have the endurance to continue this 35-year-long march. If we are able to bring young people into the fold and empower them to play a leadership role to help us to get to the end of this journey, then my optimism is further bolstered.

Nonetheless, I qualify my optimism as 'cautious' because I worry greatly about dwindling resources, waning political will and an unsettling sense of complacency that can gum up the cogs and lead to inertia that jeopardizes our efforts to end AIDS as a public health threat by 2030 . I am hopeful nonetheless that I will witness the fulfillment of this objective within my lifetime. I hope to be able to say to my grandchildren (perhaps members of the first AIDS-free generation) that we did everything that we could possibly do, that we did not delay, that we recognized the challenge of our time, that we rose to the occasion and, that because of our collective efforts, while we may not have saved every life, we saved countless millions and, possibly, billions of lives.

\section{Disclaimer}

The opinions expressed in this interview are those of the interviewee and do not necessarily reflect the views of Future Medicine Ltd.

\section{Financial \& competing interests disclosure}

The author has no relevant affiliations or financial involvement with any organization or entity with a financial interest in or financial conflict with the subject matter or materials discussed in the manuscript. This includes employment, consultancies, honoraria, stock ownership or options, expert testimony, grants or patents received or pending, or royalties.

No writing assistance was utilized in the production of this manuscript.

\section{References}

1 International Association of Providers of AIDS Care.

www.fast-trackcities.org

2 UNAIDS. 90-90-90: an Ambitious Treatment

Target to Help End the AIDS Epidemic.

UNAIDS, Geneva, Switzerland (2014). adults and adolescents. J. Int. Assoc. Provid. AIDS Care 14(Suppl. 1), S3-S34 (2015).

4 International Association of Providers of AIDS Care.

www.iapacafrohub.org.za
5 UNAIDS. Fast-Track: Ending the AIDS Epidemic by 2030. UNAIDS, Geneva, Switzerland (2014).

6 United Nations. Political Declaration on Ending AIDS. United Nations, New York, NY, USA (2016). 